\title{
AN APPLICATION OF HOMOLOGICAL ALGEBRA TO THE HOMOTOPY CLASSIFICATION OF TWO DIMENSIONAL CW-COMPLEXES
}

\author{
BY
}

MICHEAL N. DYER

\begin{abstract}
Let $\pi$ be $Z_{m} \times Z_{n}$. In this paper the homotopy types of finite connected two dimensional CW-complexes with fundamental group $\pi$ are shown to depend only on the Euler characteristic. The basic method is to study the structure of the group $\operatorname{Ext}_{Z \pi}^{1}\left(I \pi^{2}, Z\right)$ as a principal $\operatorname{End}\left(I \pi^{2}\right)$-module.
\end{abstract}

1. In this paper $\pi$ will denote the noncyclic group $Z_{m} \times Z_{n}$, which is the product of two finite cyclic groups $Z_{m}$ and $Z_{n}$. Thus the $\operatorname{gcd}(m, n) \neq 1$. For convenience, we will always assume that $m$ divides $n$. This is no restriction.

Let $X_{\Phi}$ denote the two dimensional $\mathrm{CW}$-complex modeled on the presentation $\mathscr{P}=\left\{x, y: x^{m}, y^{n},[x, y]\right\}$ of $\pi$ and let $\pi_{2}=\pi_{2} X_{\mathscr{P}} . X_{\mathscr{P}}$ is called the standard model and $\pi_{2}$ the standard module. The study of this $\pi$-module $\pi_{2}$ forms the basis of this paper.

For any $\theta \in$ Aut $\pi$ and any $\pi$-module $M$, the module ${ }_{\theta} M$ has action given by $g * m=\theta(g) m$ for any $m \in M, g \in \pi$. Two modules $M, N$ are said to be $\theta$-isomorphic iff there is an isomorphism $\alpha: M \rightarrow_{\theta} N$. The module $\pi_{2}$ splits as a short exact sequence $Z \longrightarrow \pi_{2} \rightarrow(I \pi)^{2}$ where $Z$ is the trivial $\pi$-module and $I \pi$ is the augmentation ideal in $Z \pi$. By studying the group $\operatorname{Ext}\left(I \pi^{2}, Z\right)$ we prove the following crucial theorem.

TheOREM A. For any $\pi$-module $M$ such that $M \oplus Z \pi \cong \pi_{2} \oplus Z \pi$, we have $M \cong{ }_{\theta} \pi_{2}$ for some $\theta \in$ Aut $\pi$.

Hence, $M$ is stably isomorphic to $\pi_{2}$ iff $M$ is $\theta$-isomorphic to $\pi_{2}$ for some $\theta \in$ Aut $\pi$.

The group $H^{3}\left(\pi ; \pi_{2}\right)$ is isomorphic to the cyclic group $Z_{m n}\left[D_{1}, \S 2\right]$; to each integer $q$ prime to $m n$, there is a projective ideal $(q, N) \subset Z \pi$ generated by $q$ and $N=\left(\sum_{i=1}^{m} x^{i}\right)\left(\sum_{j=1}^{n} y^{i}\right)$. The function $\partial: Z_{m n}^{*} \rightarrow \tilde{K}_{0} Z \pi$ given by $\partial(q+(m n))=$ $\{(q, N)\} \in \tilde{K}_{0} Z \pi$ is a homomorphism. A $\theta$-isomorphism $\alpha: \pi_{2} \rightarrow_{\theta} \pi_{2}$ has degree $k \in Z_{m n}^{*}$ iff $\left(\theta^{*}\right)^{-1} \alpha_{*}(1)=k$ in the diagram:

$$
H^{3}\left(\pi ; \pi_{2}\right) \stackrel{\alpha_{*}}{\rightarrow} H^{3}\left(\pi ; \pi_{\theta} \pi_{2} \stackrel{\theta^{*}}{\leftarrow} H^{3}\left(\pi ; \pi_{2}\right) .\right.
$$

TheOREM B. For any $k \in \operatorname{ker} \partial \subset Z_{m n}^{*}$ there is $a \theta \in$ Aut $\pi$ and $a \theta$-isomorphism $\alpha: \pi_{2} \rightarrow_{\theta} \pi_{2}$ of degree $k$. 
We prove Theorem $\mathrm{A}$ in $\$ 4$ and Theorem $\mathrm{B}$ in $\$ 5$.

The following Corollaries 1 and 2 follow from $A$ and $B$ just as in $\left[D_{2}\right.$, Theorem 5.5].

Definition. A $(G, 2)$-complex is a finite, connected, 2-dimensional $\mathrm{CW}$-complex having fundamental group isomorphic to $G$.

Corollary 1. Any two $\left(Z_{m} \times Z_{n}, 2\right)$-complexes have the same homotopy type iff they have the same Euler characteristic.

In the language of $\left[D_{2}\right]$, the homotopy trees $\operatorname{HT}\left(Z_{m} \times Z_{n}, 2\right)$ have essential height zero.

COROllary 2. Let $X$ be a $C W$-complex with fundamental group isomorphic to $Z_{m} \times Z_{n}$ and suppose that $X$ is dominated by a $(G, 2)$-complex. Then $X$ has the homotopy type of a $\left(Z_{m} \times Z_{n}, 2\right)$-complex iff the Wall obstruction vanishes.

In the homotopy classification of $G$-complexes for $G$ finite abelian, these results fill in a gap that existed between $G$ cyclic $\left[D_{1}\right]$ and $G$ having more than two torsion coefficients [SD]. A technique similar to this may be decisive in determining the isomorphism and $\theta$-isomorphism classes of the minimal $(G, 2)$-modules detected in [SD], for $G$ finite abelian.

2. A study of $\operatorname{Ext}\left((I \pi)^{2}, Z\right)$. By looking at the cellular chain complex of the universal cover $\tilde{X}_{\mathscr{\rho}}$ of the standard model $X_{\mathscr{\rho}}$, we may identify $\pi_{2}$ as the kernel of the following exact sequence:

$$
\varrho_{*}\left(\tilde{X}_{(\mathrm{p})}\right): \pi_{2} \longrightarrow(Z \pi)^{3} \frac{\left[\begin{array}{ccc}
N_{x} & 1-y & 0 \\
0 & x-1 & N_{y}
\end{array}\right]}{\partial_{2}}(Z \pi)^{2} \frac{(x-1, y-1)}{\partial_{1}} Z \pi \stackrel{\epsilon}{\longrightarrow} Z .
$$

For an integer $r>0$ and $z \in \pi$, let $\langle z, r\rangle=1+z+\cdots+z^{r-1}$. Then $N_{x}=\langle x, m\rangle$ and $N_{y}=\langle y, n\rangle$. The map $\varepsilon: Z \pi \rightarrow Z$ is the augmentation homomorphism. It is easy to see that $\pi_{2}$ has generators the columns of the matrix

$$
\left[\begin{array}{cccc}
x-1 & y-1 & 0 & 0 \\
0 & N_{x} & -N_{y} & 0 \\
0 & 0 & x-1 & y-1
\end{array}\right]
$$

Label the columns $g_{1}, g_{2}, g_{3}$, and $g_{4}$ respectively.

Let $\eta_{13}:(Z \pi)^{3} \rightarrow(Z \pi)^{2}$ denote the projection on the first and third coordinates. $\eta=\eta_{13} \mid \pi_{2}$ has image $I \pi^{2}$ and kernel $\pi_{2}^{\pi}=\left\{\alpha \in \pi_{2} \mid g \alpha=\alpha\right.$ for all $\left.g \in \pi\right\}=Z=$ $Z \pi(0, N, 0)$, where $N=\langle x, m\rangle\langle y, n\rangle$. Thus the extension class [E] of the extension

$$
\varepsilon: Z \succ \pi_{2} \stackrel{\eta}{\rightarrow} I \pi^{2}
$$

is a member of $E=\operatorname{Ext}_{Z \pi}^{1}\left(I \pi^{2}, Z\right)$. Sometimes, we will denote the class of the extension $\mathscr{F}: Z \gg M \rightarrow I \pi^{2}$ by $\left[\mathscr{F}_{M}\right]$. Using the fact that $\operatorname{Ext}_{Z_{\pi}}^{1}(I \pi, Z) \simeq$ $H^{2}(\pi ; Z) \cong \operatorname{Ext}_{Z}(\pi, Z) \cong \pi$, we see that $\operatorname{Ext}\left(I \pi^{2}, Z\right) \cong \pi^{2}$. We will think of $E$ as 
$2 \times 2$ matrices

$$
E=\left[\begin{array}{ll}
Z_{n} & Z_{n} \\
Z_{m} & Z_{m}
\end{array}\right] .
$$

$E$ may be considered as a right module over the ring $\operatorname{End}(I \pi)^{2}$ as follows: to each $\alpha \in \operatorname{End}(I \pi)^{2}$ and each extension class $[\mathscr{F}] \in E$ we associate the extension class $[\mathscr{F} \alpha$ ] which is the pull-back of $M$ by $\alpha$. Thus

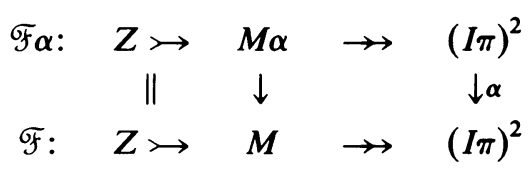

In fact, with this action, $E$ becomes a principal $\operatorname{End}(I \pi)^{2}$-module with generator [E] . To see this, we use the long exact sequence for $\operatorname{Ext}_{Z \pi}^{i}$ associated with $\mathcal{E}$ [HS, p. 139]:

$$
\operatorname{Hom}\left(I \pi^{2}, \pi_{2}\right) \rightarrow \operatorname{End}(I \pi)^{2} \stackrel{\partial}{\rightarrow} \operatorname{Ext}\left(I \pi^{2}, Z\right) \rightarrow \operatorname{Ext}\left(I \pi^{2}, \pi_{2}\right) \rightarrow \ldots
$$

The boundary operator $\partial$ is described by $\partial(\alpha)=[\mathcal{E} \alpha]$. Sometimes, when the basic extension is clear, $\partial(\alpha)$ will be denoted by $[\alpha]$. But, by using the exact sequence 2.1 , $\operatorname{Ext}_{Z \pi}^{1}\left((I \pi)^{2}, \pi_{2}\right) \cong \operatorname{Ext}_{Z \pi}^{2}\left(Z^{2}, \pi_{2}\right) \cong\left[H^{2}\left(\pi ; \pi_{2}\right)\right]^{2}=0\left[D_{1}\right.$, Lemma 6.7]. Thus we have proved the following lemma.

2.2 Lemma. $\operatorname{Ext}\left((I \pi)^{2}, Z\right)$ is a principal $\operatorname{End}(I \pi)^{2}$-module with generator [E].

Definition. Let $\mathcal{G}(E)=\left\{[\mathscr{F}] \in \|[\mathscr{F}]\right.$ is a generator of $E$ as an $\operatorname{End}(I \pi)^{2}$ module\}.

2.3 LeMMA. Suppose that $M$ is stably isomorphic to the standard module $\pi_{2}$; i.e., $M \oplus Z \pi \cong \pi_{2} \oplus Z \pi$. Then, if $M_{\pi}=M / M^{\pi}$, the extension $\mathscr{F}_{M}: Z=M^{\pi} \succ$ $M \rightarrow M_{\pi} \cong I \pi^{2}$ generates $E$ as an $\operatorname{End}\left((I \pi)^{2}\right)$-module.

Proof. If we can show that $M$ is an extension of $Z=M^{\pi}$ by $I \pi^{2}$, then $\left[\mathscr{F}_{M}\right] \in \mathcal{G}(E)$ follows using the argument above (with the exact sequence $\mathscr{F}_{M}$ ) together with the fact that

$$
H^{2}(\pi, M) \cong H^{2}(\pi ; M \oplus Z \pi) \cong H^{2}\left(\pi ; \pi_{2} \oplus Z \pi\right) \cong H^{2}\left(\pi ; \pi_{2}\right)=0
$$

since $H^{2}(\pi ; Z \pi)=0$ for any finite group [CE, p. 233]. To prove the first statement, observe that

$$
M \oplus Z \pi \cong \pi_{2} \oplus Z \pi \Rightarrow M_{\pi} \oplus Z \pi /(N) \cong\left(\pi_{2}\right)_{\pi} \oplus Z \pi /(N) .
$$

A careful, but elementary argument shows then that $M_{\pi} \oplus I \pi \simeq\left(\pi_{2}\right)_{\pi} \oplus I \pi$. Because $I \pi$ (and hence $(I \pi)^{2}$ ) satisfies the Eichler condition [SE, p. 176] and $I \pi$ is a direct summand of $\left(\pi_{2}\right)_{\pi} \cong(I \pi)^{2}$, we have, using Jacobinski's cancellation theorem [SE, Theorem 19.8], that $M_{\pi} \cong I \pi^{2}$.

NoTE. For any $(\pi, 2)$-complex $Y$, and any isomorphism $\alpha: \pi \rightarrow \pi_{1} Y$ it follows that ${ }_{\alpha} \pi_{2}(Y) \oplus Z \pi \cong \pi_{2} \oplus Z \pi$. These modules are therefore of topological interest. We will show in Theorem 4.2 that the converse is also true; that is,

$[\mathcal{F}] \in \mathcal{G}(E)$ implies that $M$ is stably isomorphic to $\pi_{2}$. 
We identify the ring of endomorphisms of $\pi(=$ End $\pi)$ as a subset of $E$.

NotATion. For each integer $a$, let $\bar{a}_{k}$ be the residue class of $a(\bmod k)$.

Let

$$
\text { End } \pi=\left\{\alpha=\left[\begin{array}{ll}
\bar{a}_{n} & \bar{b}_{n} \\
\bar{c}_{m} & \bar{d}_{m}
\end{array}\right] \in E=\left[\begin{array}{ll}
Z_{n} & Z_{n} \\
Z_{m} & Z_{m}
\end{array}\right] \mid n \text { divides } b m\right\} \text {. }
$$

Multiplication of two elements in $E$ (as $2 \times 2$-matrices) is well defined iff they are in End $\pi$. Aut $\pi \subset \mathcal{G}(E)$ is the subset of End $\pi \subset E$ consisting of invertible elements. Note that $\alpha\left(x^{i} y^{i}\right)=x^{c j+d i y^{a j+b i}}$ can be computed from

$$
\left[\begin{array}{ll}
a & b \\
c & d
\end{array}\right]\left(\begin{array}{l}
j \\
i
\end{array}\right)=\left(\begin{array}{l}
a j+b i \\
c j+d i
\end{array}\right)
$$

(observe that we have interchanged $x$ and $y$ ).

In general $\mathcal{G}(E)$ is bigger than Aut $\pi$, as $\mathcal{G}(E)$ contains the image of $\mathrm{GL}(2, Z)$ in $E$. For example,

$$
\left[\left(\begin{array}{ll}
1 & 1 \\
2 & 1
\end{array}\right)\right]=\left(\begin{array}{cc}
1 & 1 \\
\overline{2}_{m} & 1
\end{array}\right)
$$

is always in $\mathcal{G}(E)$, but never in Aut $\pi$.

2.4 LEMMA. The boundary operator $\partial: \operatorname{End}(I \pi)^{2} \rightarrow E$ is described by carrying each

$$
\alpha=\left[\begin{array}{ll}
\alpha_{11} & \alpha_{12} \\
\alpha_{21} & \alpha_{22}
\end{array}\right] \quad\left(\alpha_{i j} \in Z \pi\right)
$$

to

$$
\left[\begin{array}{ll}
\overline{\varepsilon\left(\alpha_{11}\right)_{n}} & \overline{\varepsilon\left(\alpha_{12}\right)_{n}} \\
\overline{\varepsilon\left(\alpha_{21}\right)_{m}} & \overline{\varepsilon\left(\alpha_{22}\right)_{m}}
\end{array}\right]
$$

Proof. We are thinking of $E$ as $\operatorname{End}(I \pi)^{2} / B$, where

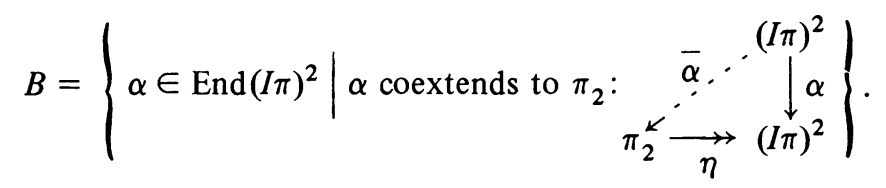

$B$ is always a right ideal, but it is not a left ideal unless $m=n$. The identification of $E$ with $\pi^{2}$ is accomplished as follows: Identify each element $\alpha$ with a $2 \times 2$ matrix

$$
\alpha=\left[\begin{array}{ll}
\alpha_{11} & \alpha_{12} \\
\alpha_{21} & \alpha_{22}
\end{array}\right]
$$

where each $\alpha_{i j} \in Z \pi$. This can be done because End $I \pi \simeq Z \pi /(N)$ for any finite group $\pi$. By direct computation one may show that any map $\beta=\left(\beta_{i j}\right)$ coextends, provided each $\beta_{i j} \in I \pi$. One simply shows directly that, if $E^{i j}(i, j=1,2)$ denotes the elementary $2 \times 2$ matrix with a one in the $i j$ th slot and zeros elsewhere, then $(x-1) E^{i j}$ and $(y-1) E^{i j}$ coextend. The $\beta$ given above is a linear combination of $(x-1) E^{i j}$ and $(y-1) E^{i j}$ (because each $\beta_{i j}=\beta_{i j}^{\prime}(x-1)+\beta_{i j}^{\prime \prime}(y-1)$ ), and hence 
coextends. For example, $\beta=\left(\begin{array}{cc}0 & 0 \\ x-1 & 0\end{array}\right)$ coextends by the map $\bar{\beta}:(I \pi)^{2} \rightarrow \pi_{2}$ given by defining $\bar{\beta}(x-1,0)=(x-1)\left(0,-N_{y}, x-1\right), \quad \beta(y-1,0)=$ $(y-1)(0,0, x-1)$, and $\bar{\beta}(0, y-1)=0=\bar{\beta}(0, x-1)$. Then $\eta \circ \bar{\beta}=\beta$ and we are done provided $\bar{\beta}$ is well defined. Using 2.1 , we identify $\operatorname{Hom}_{Z \pi}(I \pi, M)$ with $\{\alpha$ : $\left.(Z \pi)^{2} \rightarrow M: \alpha \mid \operatorname{im} \partial_{2}=0\right\}$. It is easy to check that the map $\bar{\alpha}:(Z \pi)^{2} \rightarrow \pi_{2}$ which sends $(1,0) \rightarrow\left(0,-(x-1) N_{y},(x-1)^{2}\right)$ and $(0,1) \rightarrow(0,0,(y-1)(x-1))$ is zero when restricted to im $\partial_{2}$. Then $\bar{\beta}=(\bar{\alpha}, 0):(I \pi)^{2} \rightarrow \pi_{2}$.

Thus each $\alpha=\left(\alpha_{i j}\right)$ in $\operatorname{End}(I \pi)^{2}$ is equivalent $\bmod B$ to the map $\left(\varepsilon\left(\alpha_{i j}\right)\right)$ with integer entries. One may further show that the matrices $n E^{1 j}$ and $m E^{2 j}$ coextend $(j=1,2)$. For example, $\langle y, n\rangle E^{11}$ coextends via a map $(I \pi)^{2} \rightarrow \pi_{2}$ defined by carrying $(x-1,0) \mapsto((x-1)\langle y, n\rangle, 0,0)$ and $(y-1,0),(0, x-1),(0, y-1)$ all to zero. Thus we see that the map $\partial: \operatorname{End}(I \pi)^{2} \rightarrow \operatorname{Ext}\left((I \pi)^{2}, Z\right)$ can be described by

$$
\left[\begin{array}{ll}
\alpha_{11} & \alpha_{12} \\
\alpha_{21} & \alpha_{22}
\end{array}\right] \mapsto\left[\begin{array}{ll}
\overline{\varepsilon\left(\alpha_{11}\right)_{n}} & \overline{\varepsilon\left(\alpha_{12}\right)_{n}} \\
\overline{\varepsilon\left(\alpha_{21}\right)_{m}} & \overline{\varepsilon\left(\alpha_{22}\right)_{m}}
\end{array}\right]
$$

We will isolate several subsets of $\mathcal{G}(E)$, which we then proceed to study. Let

$$
\begin{aligned}
S \mathcal{G}(E) & =\left\{\left[\mathscr{F}_{M}\right] \in E \mid M \oplus Z \pi \cong \pi_{2} \oplus Z \pi\right\}, \\
{ }_{\theta} \text { Iso } & =\left\{\left[\mathscr{F}_{M}\right] \in E \mid M \cong{ }_{\theta} \pi_{2} \text { for some } \theta \in \text { Aut } \pi\right\}, \text { and } \\
\text { Iso } & =\left\{\left[\mathscr{F}_{M}\right] \in E \mid M \cong \pi_{2}\right\} .
\end{aligned}
$$

Clearly, by 2.3 , any module $M$ stably isomorphic to $\pi_{2}$ is already an extension of $Z$ by $I \pi^{2}$.

The following inclusions hold:

$$
\mathcal{G}(E) \supset S \mathcal{G}(E) \supset{ }_{\theta} \text { Iso } \supset \text { Iso. }
$$

The first inclusion follows from Lemma 2.3. The last inclusion is clear; the second follows as any module $M$ which is $\theta$-isomorphic to $\pi_{2}$ may be embedded in the sequence: $0 \rightarrow M \rightarrow{ }_{\theta} C_{2} \rightarrow{ }_{\theta} C_{1} \rightarrow{ }_{\theta} Z \pi \rightarrow Z$; thus $M$ is stably isomorphic to $\pi_{2}$ by Schanuel's lemma.

One can show that $\mathcal{G}(E)=\left\{\left[\mathscr{F}_{M}\right] \in E \mid M\right.$ has the same genus as $\left.\pi_{2}\right\}$. However, it is not true that any module $M$ of the same genus as $\pi_{2}$ is an extension of $Z$ by $I \pi^{2}$.

For future reference, we record the following easily proved characterization of Iso.

2.5 Proposition. Let $\left[\mathscr{F}_{M}\right] \in \mathcal{G}(E)$. Then $M \cong \pi_{2}$ iff $[\mathscr{F}]=[\mathcal{E} \alpha] \in E$ for some $\alpha \in \mathrm{GL}(2, Z \pi /(N)) \subset \operatorname{End}(I \pi)^{2}$.

3. Comparison of $\pi_{2}$ and $\pi_{2} \theta$. In this section we compare the pullback $\pi_{2} \theta$ and the module ${ }_{\theta} \pi_{2}$ for any $\theta \in$ Aut $\pi$. We continue the assumption that $m \mid n$.

Let ${ }_{\theta} \mathcal{E}$ denote the extension $Z \longrightarrow_{\theta} \pi_{2} \rightarrow{ }_{\theta} I \pi^{2} \approx I \pi^{2}$, with the isomorphism given by $\left[\begin{array}{cc}Z \theta^{-1} \\ 0\end{array} \boldsymbol{z}^{-1}\right]$. Recall that any $\boldsymbol{\theta} \in$ Aut $\pi$ can be written as a product $\boldsymbol{\theta}=$ $E_{1} E_{2} \ldots E_{k} D$ where each $E_{i}=\left[\begin{array}{ll}1 & a_{i} \\ 0 & 1\end{array}\right]$ or $\left[\begin{array}{ll}1 & 0 \\ b_{i} & 0\end{array}\right]\left(a_{i}, b_{i} \in Z\right.$ and $n$ divides $m_{i}, i=$ $1, \ldots, k)$ is an elementary automorphism and $D=\left[\begin{array}{ll}p & 0 \\ 0\end{array}\right](p, q \in Z)$ is a diagonal automorphism, provided $p$ is prime to $n$ and $q$ is prime to $m$ [S, Proposition 6]. 
Definition. If $\theta=\left[\begin{array}{cc}1 & -r \\ 0 & 1\end{array}\right]$ (respectively, $\left.\left[\begin{array}{cc}1 & 0 \\ -s & 1\end{array}\right],\left[\begin{array}{ll}p & 0 \\ 0 & q\end{array}\right]\right)$ is a member of Aut $\pi$ (i.e., $m r$ is divisible by $n$ ) then let

$$
\theta^{0}=\left[\begin{array}{cc}
1 & 0 \\
r m / n & 1
\end{array}\right] \quad\left(\operatorname{respectively}\left[\begin{array}{cc}
1 & s n / m \\
0 & 1
\end{array}\right],\left[\begin{array}{ll}
p & 0 \\
0 & q
\end{array}\right]\right) .
$$

Then, for any $\theta \in$ Aut $\pi, \theta^{0}$ is the element of Aut $\pi$ defined by writing $\theta=$ $E_{1} \ldots E_{k}\left[\begin{array}{ll}p & 0 \\ 0 & q\end{array}\right]$, where $E_{1}, \ldots, E_{k}$ are elementary automorphisms, and setting $\theta^{0}=$ $\theta_{1}^{0} \cdots \theta_{k}^{0}\left[\begin{array}{l}p \\ 0\end{array}\right]$. Notice that $\left(\theta^{0}\right)^{0}=\theta$.

3.1 Theorem. For any $\theta \in$ Aut $\pi,\left[{ }_{\theta} \mathcal{E}\right]=\left[\mathcal{E} \theta^{0}\right]$ and $[\mathcal{E} \theta]=\left[\theta_{\theta} \mathcal{E}\right]$.

Proof. Let $\theta \in$ Aut $\pi$ be written as a product of a diagonal automorphism $D=\left[\begin{array}{ll}p & 0 \\ 0\end{array}\right]$ and a product $E_{1} \ldots E_{k}$ of elementary automorphisms. The theorem will follow from the fact that $[\mathcal{E}(\alpha \beta)]=[(\mathcal{E} \alpha) \beta]$ and ${ }_{(\alpha \beta)} \pi_{2}={ }_{\beta}\left({ }_{\alpha} \pi_{2}\right)$ provided we can show that the theorem is true for $D$ and $E_{i}$. We will give only the proof for $\theta=\left[\begin{array}{cc}1 & 0 \\ -r & 1\end{array}\right]\left(\right.$ i.e. $\left.\theta(x)=x, \theta(y)=x^{-r} y\right)$, as the others are similar.

3.2 LeMMA. Let $\pi=Z_{m} \times Z_{n}$, with $m \mid n$, and $\theta=\left[\begin{array}{cc}1 & 0 \\ -r & 1\end{array}\right], \theta^{0}=\left[\begin{array}{cc}1 & m / m \\ 0 & 1\end{array}\right]$. Then $\left[{ }_{\theta} \mathcal{E}\right]=\left[\mathcal{E} \theta^{0}\right]$ in $\operatorname{Ext}\left(I \pi^{2}, Z\right)$.

Proof. Let $\beta=\sum_{i=1}^{n} y^{i-1}\langle x, r i\rangle, k=r n / m$, and $\bar{\theta}=\theta^{-1}=\left[\begin{array}{ll}1 & 0 \\ r & 1\end{array}\right]$. Straightforward calculation using 2.1 shows that the matrix

$$
\left[\begin{array}{ccc}
1 & 0 & k \\
0 & x^{r} & \beta \\
0 & 0 & 1
\end{array}\right]
$$

defines a map from $C_{2}=Z \pi e_{1} \oplus Z \pi e_{2} \oplus Z \pi e_{2} \rightarrow{ }_{\theta} C_{2}={ }_{\theta} Z \pi e_{1} \oplus{ }_{\sigma_{\theta}} Z \pi e_{2} \oplus{ }_{\theta} Z \pi e_{3}$ (i.e., $e_{1} \rightarrow(1,0,0), e_{2} \rightarrow\left(0, x^{r}, 0\right), e_{3} \rightarrow(k, \beta, 1)$ and extend linearly) which sends $\pi_{2}$ $\left(\subset C_{2}\right)$ into ${ }_{\theta} \pi_{2}\left(\subset{ }_{\bar{\theta}} C_{2}\right)$. This same matrix defines a map from ${ }_{\theta} C_{2} \rightarrow{ }_{\theta}\left({ }_{\theta} C_{2}\right)=C_{2}$ and hence a map $\theta_{2}:{ }_{\theta} \pi_{2} \rightarrow \pi_{2}$. On the generators for ${ }_{\theta} \pi_{2}, \theta_{2}$ looks like

$$
\begin{aligned}
\bar{\theta}(x-1) *{ }_{\theta} e_{1} & =(x-1) e_{1} \\
& \mapsto \bar{\theta}(x-1) e_{1}, \bar{\theta}(y-1) *{ }_{\theta} e_{1}+\bar{\theta}\left(N_{x}\right) *{ }_{\theta} e_{2}=\left(y-1, N_{x}, 0\right) \\
& \mapsto \bar{\theta}(y-1) e_{1}+x^{r} \bar{\theta}\left(N_{x}\right) e_{2}, \bar{\theta}\left(-N_{y}\right) *{ }_{\theta} e_{2}+\bar{\theta}(x-1) *{ }_{\theta} e_{3} \\
& =\left(0,-N_{y}, x-1\right) \\
& \mapsto k \bar{\theta}(x-1) e_{1}+\left(-x^{r} \bar{\theta}\left(N_{y}\right)+\bar{\theta}(x-1) \beta\right) e_{2}+\bar{\theta}(x-1) e_{3}
\end{aligned}
$$

and finally,

$$
\bar{\theta}(y-1) *{ }_{\theta} e_{3}=(y-1) e_{3} \mapsto k \bar{\theta}(y-1) e_{1}+\beta \bar{\theta}(y-1) e_{2}+\bar{\theta}(y-1) e_{3} .
$$

Inspection shows that $\theta_{2}$ induces a map $\theta_{2}^{\prime}:{ }_{\theta}(I \pi)^{2} \rightarrow(I \pi)^{2}$ with matrix $\left[\begin{array}{ll}1 & k \\ 0 & 1\end{array}\right]$. Here, 1 : ${ }_{\theta} I \pi \rightarrow I \pi$ is the map induced by $Z \bar{\theta}:{ }_{\theta} Z \pi \rightarrow Z \pi$. Let $Z \theta$ denote the isomorphism $I \pi \rightarrow{ }_{\theta} I \pi$ induced from $Z \theta: Z \pi \rightarrow{ }_{\theta} Z \pi$. The composition

$$
(I \pi)^{2} \stackrel{\left(\begin{array}{cc}
Z \theta & 0 \\
0 & Z \theta
\end{array}\right)}{\rightarrow}(I \pi)^{2} \stackrel{\theta_{2}^{\prime}}{\rightarrow}(I \pi)^{2}
$$

yields a map with matrix $\left[\begin{array}{ll}1 & k \\ 0 & 1\end{array}\right]$. 
4. Characterizing $\mathcal{S}(E)$. We will now characterize the set $\mathcal{G}(E)$ of generators of $E=\operatorname{Ext}\left(I \pi^{2}, Z\right)$. For each $\alpha=\left(\alpha_{i j}\right) \in \operatorname{End}(I \pi)^{2}\left(\alpha_{i j} \in Z \pi\right)$, let $\varepsilon(\alpha)$ denote the integer matrix with entries $\varepsilon\left(\alpha_{i j}\right)(i, j=1,2)$. lent:

4.1 Proposition. Let $\alpha=\left(\alpha_{i j}\right) \in \operatorname{End}(I \pi)^{2}\left(\alpha_{i j} \in Z \pi\right)$. The following are equiva-

(a) $[\alpha] \in \mathcal{G}(E)$.

(b) $\exists \alpha^{\prime} \in \operatorname{End}(I \pi)^{2}$ such that $\alpha \alpha^{\prime}-1 \in B$.

(c) The determinant of $\varepsilon(\alpha)$ is prime to $m$ and there are integers $s, t$ such that $\varepsilon\left(\alpha_{11}\right) s+\varepsilon\left(\alpha_{12}\right) t \equiv 1(\bmod n)$.

(d) There is an integer matrix $\gamma=\left[\begin{array}{ll}a & b \\ c & d\end{array}\right]$ such that

$$
\bar{\gamma}_{n}=\left[\begin{array}{cc}
\bar{a}_{n} & \bar{b}_{n} \\
\bar{c}_{n} & \bar{d}_{n}
\end{array}\right] \in \mathrm{GL}\left(2, Z_{n}\right)
$$

and $[\alpha]=[\gamma] \in E$.

Proof. (a) $\Rightarrow$ (b). If $[\mathcal{E} \alpha] \in \mathcal{G}(E)$, then [E] itself must be a pullback of $Z$ $\succ \pi_{2} \alpha \rightarrow I \pi^{2}$ by some $\alpha^{\prime}: I \pi^{2} \rightarrow I \pi^{2}$. Thus [E] $=\left[\mathcal{E} \alpha \alpha^{\prime}\right]$ implies $\alpha \alpha^{\prime}-1 \in B$.

(b) $\Rightarrow$ (c). It is easy to see that the correspondence $[\alpha] \in E \mapsto \operatorname{det} \varepsilon(\alpha) \in Z$ is well defined modulo $m$. $\alpha \alpha^{\prime}-1 \in B$ implies $\operatorname{det} \varepsilon(\alpha) \cdot \operatorname{det} \varepsilon\left(\alpha^{\prime}\right) \equiv 1(\bmod m)$. Thus det $\varepsilon(\alpha)$ is prime to $m$. Furthermore, $\varepsilon\left(\alpha_{11}\right) \varepsilon\left(\alpha_{11}^{\prime}\right)+\varepsilon\left(\alpha_{12}\right) \varepsilon\left(\alpha_{21}^{\prime}\right)-1 \equiv 0$ $(\bmod n)$ follows by looking at the 11-coordinate of $\partial\left(\alpha \alpha^{\prime}-1\right)$.

(c) $\Rightarrow(\mathrm{d})$. Because $m$ divides $n$, the natural map $\bar{a}_{n} \mapsto \bar{a}_{m}(a \in Z)$ induces a surjection $Z_{n}^{*} \rightarrow Z_{m}^{*}$. Let $d=\operatorname{det} \varepsilon(\alpha) . d$ is prime to $m$, so there is an integer $l$ such that $d+l m$ is prime to $n$. $s \varepsilon\left(\alpha_{11}\right)+t \varepsilon\left(\alpha_{12}\right)=1+k n$ then yields $d+l(1+k n) m$ is prime to $n$. Now consider the integer matrix

$$
\gamma=\left[\begin{array}{cc}
\varepsilon\left(\alpha_{11}\right) & \varepsilon\left(\alpha_{12}\right) \\
\varepsilon\left(\alpha_{21}\right)-l \text { ltm } & \varepsilon\left(\alpha_{22}\right)+l s m
\end{array}\right] .
$$

We claim that $\gamma$ represents an element of $\operatorname{GL}\left(2, Z_{n}\right)$; i.e., that det $\gamma$ is prime to $n$. Consider

$$
\operatorname{det} \gamma=d+\operatorname{lm}\left(\varepsilon\left(\alpha_{11}\right) \cdot s+\varepsilon\left(\alpha_{12}\right) \cdot t\right)=d+\operatorname{lm}(1+k n)
$$

which is prime to $n$. Clearly $[\gamma]=[\alpha]$, by Lemma 2.4 .

(d) $\Rightarrow$ (a). Choose an integer matrix $\gamma$ so that $[\gamma]=[\alpha]$ in $E$ and $\bar{\gamma}_{n} \in \operatorname{GL}\left(2, Z_{n}\right)$. Let $\gamma^{\prime}$ be an integer matrix which represents the inverse $\left(\bar{\gamma}_{n}\right)^{-1}$. Then $\overline{\left(\gamma \gamma^{\prime}-1\right)_{n}}=$ 0 implies that $\partial\left(\gamma \gamma^{\prime}-1\right)=0$. We claim that $\alpha \gamma^{\prime}-1 \in B$. Choose $b \in B$ so that $\alpha=\gamma+b$. Then $\alpha \gamma^{\prime}-1=(\gamma+b) \gamma^{\prime}-1=\gamma \gamma^{\prime}-1+b \gamma^{\prime}$. But $B$ is a right ideal, so $b \gamma^{\prime} \in B$. Hence $\alpha \gamma^{\prime}-1 \equiv \gamma \gamma^{\prime}-1 \equiv 0(\bmod B)$ and $\alpha \in \mathcal{G}(E)$.

4.2 ThEOREM. $\mathcal{G}(E)={ }_{\theta}$ Iso.

Proof. By Proposition 4.1, given any $[\alpha] \in \mathcal{G}(E)$ there is a $2 \times 2$ integer matrix $A=\left[\begin{array}{ll}a & b \\ c & d\end{array}\right]$ whose determinant is prime to $n$, and such that $[A]=[\alpha] \in E$. Consider $\bar{A}_{n}$ as the matrix $\bmod n$, that is to say, $A$ represents an element of $\operatorname{GL}\left(2, Z_{n}\right)=$ $\operatorname{Aut}\left(Z_{n} \times Z_{n}\right)$. By Proposition 6 of $[\mathrm{S}], A \equiv E_{1} E_{2} \ldots E_{k} D(\bmod n)$ where $D=$ $\left[\begin{array}{ll}p & 0 \\ 0 & q\end{array}\right]$ is a diagonal automorphism $(p, q$ are prime to $n)$ and each $E_{i}$ is an elementary 
matrix of the form $\left[\begin{array}{ll}1 & \gamma_{i} \\ 0 & 1\end{array}\right]$ or $\left[\begin{array}{ll}1 & 0 \\ \gamma_{i} & 1\end{array}\right]$, with $p, q, \gamma_{i} \in Z$. Let $M=E_{1} E_{2} \ldots E_{k} D$ be the integer matrix which is the product of $E_{1}, E_{2}, \ldots, E_{k}$, and $D$. Then

$$
\begin{aligned}
{[\delta \alpha] } & =[\mathcal{E} A]=[\mathcal{E} M] \quad(\text { because } \alpha \equiv A \equiv M \bmod B) \\
& =\left[\left(\ldots\left(\left(\mathcal{E} E_{1}\right) E_{2}\right) \cdots E_{k}\right) D\right]
\end{aligned}
$$

(because pullbacks commute with composition).

But, by Proposition 2.5

$$
\pi_{2} \cong \pi_{2} E_{1} \cong \pi_{2} E_{1} E_{2} \cong \cdots \cong \pi_{2} E_{1} \cdots E_{k}
$$

because each $E_{i} \in \operatorname{Aut}(I \pi)^{2} . D$ is a member of Aut $\pi$ implies that

$$
\pi_{2} \cong \pi_{2} E_{1} \cdots E_{k} \cong{ }_{D} \pi_{2} M \cong{ }_{D} \pi_{2} \alpha
$$

by invoking Theorem 3.1.

We have seen in $\$ 2$ that the map $\bar{\partial}=\partial \mid G L(2, Z \pi /(N)): \operatorname{GL}(2, Z \pi /(N)) \rightarrow \mathcal{G}(E)$ is onto the subset. Iso. We yould like to determine im $\bar{\partial}$. As above, each elementary automorphism $\alpha=\left[\begin{array}{ll}1 & \gamma \\ 0 & 1\end{array}\right]$ or $\left[\begin{array}{ll}1 & 0 \\ \gamma & 1\end{array}\right]$ yields $\pi_{2} \alpha \cong \pi_{2}$. The diagonal automorphisms $\beta=\left[\begin{array}{ll}p & 0 \\ 0 & q\end{array}\right](p, q \in Z)$ of $\pi$ which are clearly in im $\bar{\partial}$ have $\bar{p}_{m n}, \bar{q}_{m n} \in \operatorname{ker}\left\{\partial: Z_{m n}^{*} \rightarrow\right.$ $\left.\tilde{K}_{0} Z \pi\right\}$; for, choosing units $\bmod N u, v \in Z_{\pi} /(N)^{*}$ such that $\varepsilon(u)=p, \varepsilon(v)=q$ $[4,2.1]$, we have $\alpha=\left[\begin{array}{ll}u & 0 \\ 0 & 0\end{array}\right] \in \operatorname{GL}\left(2, Z_{\pi} /(N)\right)$ and $\bar{\partial} \alpha=\beta$. We make the following convenient hypothesis [SD, §4].

REDUCTION HYPOTHESIS. The natural projection $Z_{m n}^{*} \rightarrow Z_{n}^{*}$ remains surjective when restricted to ker $\partial$.

The reduction hypothesis is satisfied whenever $n=m=p$, an odd prime [U]. Whenever the reduction hypothesis is satisfied, the above argument implies that $\bar{\partial}$ is surjective.

4.3 Corollary. If the reduction hypothesis is true, then each $[\alpha] \in \mathcal{G}(E)$ has $\pi_{2} \alpha \cong \pi_{2} ;$ i.e., $\mathcal{G}(E)=$ Iso.

5. Shuffling $k$-invariants. Recall that $\pi=Z_{m} \times Z_{n}$, with $m \mid n$ and generators $x, y$ of order $m$ and $n$, respectively. For each $\pi$-module $M, H^{3}(\pi ; M)$ is isomorphic to $\operatorname{Hom}_{Z_{\pi}}\left(\pi_{2}, M\right) / \mathscr{B}$, where $\mathscr{B}=\left\{\beta: \pi_{2} \rightarrow M \mid \beta\right.$ extends to a map $\left.\bar{\beta}: C_{2} \rightarrow M\right\}$. For each $\alpha \in \operatorname{Hom}_{Z_{\pi}}\left(\pi_{2}, M\right)$, let $\{\alpha\}=\alpha+\mathscr{B}$ be the class of $\alpha$ in $H^{3}(\pi ; M)$. If $\theta$ is a member of Aut $\pi$, then $\theta^{*}: H^{*}\left(\pi ; \pi_{2}\right) \rightarrow H^{*}\left(\pi ;{ }_{\theta} \pi_{2}\right)$ may be computed by choosing a chain map from $e_{*}\left(\tilde{X}_{\odot}\right) \rightarrow_{\theta} \bigodot_{*}\left(\tilde{X}_{\odot}\right)$ covering the identity.

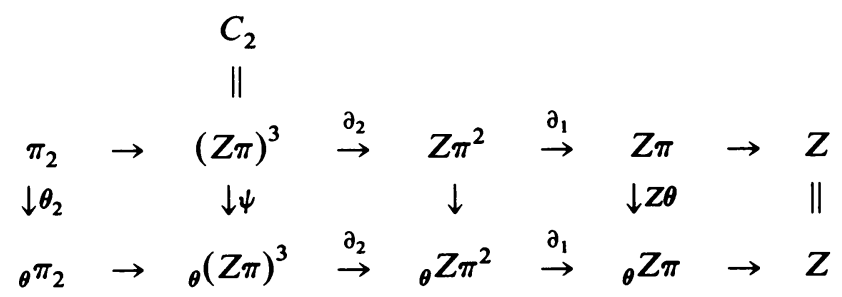

It follows that for any $\alpha \in$ End $\pi_{2}, \theta^{*}\{\alpha\}=\left\{\theta_{2} \alpha\right\} \in H^{3}\left(\pi ; \theta_{\theta}\right)$. 
If $\theta=\left[\begin{array}{ll}1 & 0 \\ 0 & p\end{array}\right]$, where $p$ is prime to $m$ (i.e., $\theta(x)=x^{p}, \theta(y)=y$ ), then

$$
\psi=\left[\begin{array}{ccc}
\langle x, p\rangle & 0 & 0 \\
0 & \langle x, p\rangle & 0 \\
0 & 0 & 1
\end{array}\right]:(Z \pi)^{3} \rightarrow{ }_{\theta}(Z \pi)^{3} \text { induces } \theta_{2}: \pi_{2} \rightarrow{ }_{\theta} \pi_{2} .
$$

The following lemma will prove useful.

5.1 LEMMA. Let $v \in Z \pi$ be a unit $(\bmod N)$ having augmentation $\varepsilon(v)=p$. Then there is a unit $(\bmod N) u \in Z \pi$ having $\varepsilon(u)=p$ and such that $u=\langle x, p\rangle+$ $(y-1) \alpha$, for some $\alpha \in Z \pi$.

Proof. For each $v \in Z \pi$, let $v_{y} \in Z\left(Z_{m}(x)\right)$ denote the image of $v$ under the map $\varepsilon_{y}: Z \pi \rightarrow Z\left(Z_{m}\right)$ obtained by setting $y=1 . v$ is a unit $\bmod N$ implies $v_{y}$ is a unit $\bmod N_{x}$, which in turn implies that $v_{y}=w\langle x, p\rangle$, where $w$ is a unit in $Z\left(Z_{m}\right)$. $w$ is also a unit in $Z \pi$ so $u=w^{-1} v$ has $u_{y}=\langle x, p\rangle$. Hence $u-\langle x, p\rangle$ is in the kernel of the map $\varepsilon_{y}$. It is easy to see that $u-\langle x, p\rangle=(y-1) \alpha$ for some $\alpha \in Z \pi$.

Recall the homomorphism $\partial: Z_{m n}^{*}\left(=\right.$ units in $\left.H^{3}\left(\pi ; \pi_{2}\right)\right) \rightarrow \tilde{K}_{0} Z_{\pi}$ given by $\partial\left(\bar{p}_{m n}\right)=\{(p, N)\} \in \tilde{K}_{0} Z \pi$. It is known that $\bar{p}_{m n} \in$ ker $\partial$ iff there is a $v \in Z \pi$ which is a unit $\bmod N$ whose augmentation $\varepsilon(v)=p,\left[\mathbf{D}_{2}, 2.1\right]$. A $\theta$-homomorphism $\alpha: \pi_{2} \rightarrow_{\theta} \pi_{2}$ has degree $k \in H^{3}\left(\pi ; \pi_{2}\right)$ iff $k\left\{\theta_{2}\right\}=\{\alpha\}$. For each $k \in \operatorname{ker} \partial$, we will construct a $\theta$-isomorphism $\pi_{2} \rightarrow_{\theta} \pi_{2}$ of degree $k$. This will prove Theorem B. We commence the proof.

Given $\bar{k}_{m n} \in \operatorname{ker} \partial \subset Z_{m n}^{*}$. Choose $u \in Z_{\pi}$ such that $\varepsilon(u)=k$ and $u$ is a unit $\bmod N . k$ is prime to $m n$ implies that $k$ is prime to $m^{2} n$. Choose an integer $p$ such that $p k+s m^{2} n=1$. Let $\theta$ be the automorphism which carries $x \rightarrow x^{p}, y \rightarrow y$. $\bar{p}_{m n}=\bar{k}_{m n}^{-1}$ in $Z_{m n}^{*}$ implies $\bar{p}_{m n} \in$ ker $\partial$. Thus there is a $v \in Z \pi$ such that $\varepsilon(v)=p, v$ is a unit $\bmod N$, and $v=\langle x, p\rangle+(y-1) \alpha$ for some $\alpha \in Z \pi$.

Let $e_{i}(i=1,2,3)$ denote the natural basis for $(Z \pi)^{3}$. Define homomorphisms $p_{i j}:(Z \pi)^{3} \rightarrow_{\theta}(Z \pi)^{3}$ sending $e_{i} \rightarrow g_{j}$ ( $g_{j}$ is a generator of $\pi_{2}$, hence, of ${ }_{\theta} \pi_{2}$, see $\$ 2$ ) and $e_{k} \rightarrow 0 \quad(k \neq i)(i=1,2,3 ; j=1,2,3,4)$. Note that $\bar{p}_{i j}=p_{i j} \mid \pi_{2}$ defines a degree 0 map from $\pi_{2} \rightarrow_{\theta} \pi_{2}$.

Consider the map $M=\psi+\alpha p_{12}:(Z \pi)^{3} \rightarrow_{\theta}(Z \pi)^{3} . M$ has matrix

$$
\left[\begin{array}{ccc}
v=\langle x, p\rangle+(y-1) \alpha & 0 & 0 \\
\alpha N_{x} & \langle x, p\rangle & 0 \\
0 & 0 & 1
\end{array}\right]
$$

$\bar{M}=\left.M\right|_{\pi_{2}}: \pi_{2} \rightarrow_{\theta} \pi_{2}$ carries $g_{1} \mapsto\left(x^{p}-1\right)(v, 0,0), g_{2} \mapsto\left(v(y-1), \alpha N_{x}(y-1)+\right.$ $\left.p N_{x}, 0\right), g_{3} \mapsto\left(0,-N_{y}\langle x, p\rangle, x^{p}-1\right)$, and $g_{4} \mapsto(0,0, y-1)$. The map $\overline{\bar{M}}:(I \pi)^{2}$

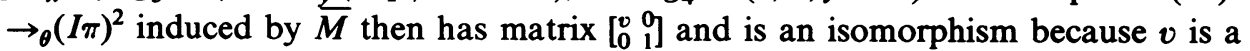
unit $(\bmod N)$.

We will now alter $\bar{M}$ to give an isomorphism $\pi_{2} \rightarrow_{\theta} \pi_{2}$ of degree $\bar{k}_{m n}$. Look at $Q=u M+s N p_{22}:(Z \pi)^{3} \rightarrow_{\theta}(Z \pi)^{3} \cdot Q$ then has matrix

$$
\left[\begin{array}{ccc}
u v & 0 & 0 \\
\alpha u N_{x} & u\langle x, p\rangle+s m N & 0 \\
0 & 0 & 0
\end{array}\right]
$$


This clearly restricts to a map $\bar{Q}: \pi_{2} \rightarrow_{\theta} \pi_{2}$ having degree $\bar{k}_{m n}$ because $\{\bar{Q}\}=\left\{u \theta_{2}\right.$ $\left.+u \alpha \bar{\varphi}_{12}+s N \bar{p}_{22}\right\}=\left\{u \theta_{2}\right\}=\left\{\varepsilon(u) \theta_{2}\right\}=k\left\{\theta_{2}\right\}$. Clearly $\bar{Q}$ induces the same map as $u \bar{M}$ on $I \pi^{2} \rightarrow_{\theta} I \pi^{2}\left(s N \bar{p}_{22}\right.$ restricted to $(I \pi)^{2}$ is zero) and is an isomorphism. We need only show that $\left.Q\right|_{\pi^{*}}$ is the identity: $Q(0, N, 0)=(u\langle x, p\rangle+\operatorname{sm} N)(0, N, 0)$ $=\left(k p+s m^{2} n\right)(0, N, 0)=(0, N, 0)$.

\section{REFERENCES}

[CE] H. Cartan and S. Eilenberg, Homological algebra, Princeton Univ. Press, Princeton, N. J., 1956. [D1] M. Dyer, Homotopy classification of $(\pi, m)$-complexes, J. Pure Appl. Algebra 7 (1976), 429-482.

$\left[\mathrm{D}_{2}\right] \ldots$, On the essential height of homotopy trees with finite fundamental group, Compositio Math. 36 (1978), 209-224.

[HS] P. Hilton and U. Stammbach, A course in homological algebra, Springer-Verlag, New York, 1970.

[S] A. Sieradski, Combinatorial isomorphisms and combinatorial homotopy equivalences, J. Pure Appl. Algebra 7 (1976), 59-95.

[SD] A. Sieradski and M. Dyer, Distinguishing arithmetic for certain stably isomorphic modules, J. Pure Appl. Algebra 15 (1979), 199-217.

[SE] R. G. Swan and E. G. Evans, $K$-theory of finite groups and orders, Lecture Notes in Math., vol. 149, Springer-Verag, Berlin and New York, 1970.

[U] S. Ullom, Non-trivial lower bounds for class groups of integral group rings, Illinois J. Math. 20 (1976), 314-329.

Department of Mathematics, University of Oregon, Eugene, Oregon 97403 